\title{
AVALIAÇÃO DE FERRAMENTAS DE VIDEOCONFERÊNCIA PARA A PLATAFORMA MOODLE
}

\author{
Maria de Fátima Webber do Prado Lima - CCET/UCS - mfwplima@ucs.br
}

Sinara Kich - CCET/UCS - sinarakich@gmail.com

Carine G. Webber - CCET/UCS - cgwebber@ucs.br

\begin{abstract}
Resumo. É importante que um ambiente virtual de aprendizagem disponibilize meios para promover a interatividade e comunicação. A videoconferência transmite mensagens não verbais de expressões faciais, de olhares, do timbre $e$ do tom da voz que são mais semelhantes com as que ocorrem na aula presencial. $O$ Moodle não possui nenhuma ferramenta de videoconferência nativa em sua plataforma. Este artigo avalia a integração das ferramentas BigBlueButton e OpenMeetings na plataforma Moodle. Para a redução de falhas no processo de decisão foi utilizado o Método Analítico Hierárquico.
\end{abstract}

Palavras chaves: Ferramentas de videoconferência, Moodle, BigBlueButton, OpenMeetings.

\section{VIDEOCONFERENCING TOOLS EVALUATION FOR MOODLE PLATFORM}

\begin{abstract}
A virtual learning environment must provide means to support communication and interaction among users. Videoconferencing tools can transmit facial non-verbal messages such as expressions, glances, the timbre and tone of voice similarly to those that occur in the presential classroom. Such an important resource is not present in Moodle Software, since it has no native video conferencing tool. In order to fulfill this gap, this article evaluates the integration of BigBlueButton and OpenMeetings tools into Moodle platform. To reduce failures in the decision process, it has been used the Analytical Hierarchical Method.
\end{abstract}

Keywords: Videoconferencing tools, Moodle,

BigBlueButton, OpenMeetings.

\section{Introdução}

Os ambientes virtuais de aprendizagem (ALAVA et al, 2002) tornaram o aprendizado mais colaborativo, eximindo do professor o papel de único responsável pelo conteúdo, tornando-o, assim, um facilitador da aprendizagem e forçando-o a conhecer novas tecnologias. Porém, o uso de um ambiente virtual por si só não garante mudanças no ensino, visto que, de fato, é preciso saber utilizá-lo. De acordo com Silva (2006), em um ambiente virtual, deve existir interação e troca de informações ao invés da mera reprodução de conteúdos por meio de oratória. Entretanto, isso não depende somente da tecnologia utilizada. Conforme Moraes (2002), para que a utilização dos AVAs seja proveitosa e, também, parte influente de uma educação de qualidade, ela deve produzir 
interesse, curiosidade, colaboração, bem como promover a busca por respostas, análise e solução de problemas.

Ferramentas como o bate-papo e fórum propiciam interação, no entanto, não são capazes de transmitir as mensagens não verbais de expressões faciais, de olhares, do timbre e do tom da voz. Muitas vezes, é mais difícil e mais demorado se expressar por meio da escrita do que se expressar pela fala, sabendo de que o comportamento das pessoas, na comunicação, pode mudar quando não são vistas. A videoconferência permite a comunicação entre várias pessoas ao mesmo tempo e em tempo real. É o recurso que propicia trocas mais semelhantes com as que ocorrem na aula presencial, pois é possível ver o professor, ouvi-lo, falar com ele e, inclusive, com os colegas.

Existem diversas plataformas de ambientes virtuais de aprendizagem disponíveis. Entre elas, destaca-se o Moodle, o qual foi projetado para fornecer a educadores, administradores e alunos a possibilidade de criar ambientes de aprendizagem personalizados em um único sistema integrado. $O$ conjunto de ferramentas que o Moodle oferece é um dos seus pontos fortes, pois se trata de um conjunto variado e extenso que trabalha comunicação, discussão, avaliação e construção coletiva, contendo, assim, diversos recursos para o trabalho dos professores. Porém o Moodle não dispõe nativamente de um recurso de videoconferência.

Este artigo apresenta uma avaliação da integração de ferramentas de videoconferência na plataforma Moodle. $\mathrm{O}$ artigo proposto está organizado em 5 seções. Inicialmente a seção 2 relaciona a plataforma moodle e as características de algumas ferramentas de videoconferência. A seção 3 descreve os testes realizados nas ferramentas BigBlueButton and OpenMeetings. A seção 4 apresenta os resultados obtidos da avaliação das ferramentas. Por fim, a seção 5 conclui a análise realizada.

\section{O Moodle e as Ferramentas de Videoconferência}

Os alunos sentem necessidade de interação por diversos motivos. Conforme pesquisa realizada na UNISUL (Universidade do Sul de Santa Catarina), os principais motivos para a busca por interação são dúvidas operacionais, de conteúdo e de avaliação, iniciativas para a colaboração, desejo de afiliação ao grupo, manifestações de afetividade, negociação e conflitos e, também, desejo de privacidade e não supervisão (MÜLBERT et al., 2011). Outra pesquisa, realizada em uma universidade portuguesa sobre a experiência do Ensino a Distância com alunos de um curso de extensão, aponta que $87 \%$ dos alunos afirmam que a interação com os colegas foi algo fundamental para a realização do curso (MACHADO et al., 2005).

Na educação a distância, uma grande questão é como atender a necessidade de interação. Nessa perspectiva, a videoconferência, em ambientes virtuais de aprendizagem, tem o propósito de ser uma alternativa para sanar essa demanda. Moran (1998) defende que é parte do processo de educação ajudar a desenvolver habilidades para se comunicar efetivamente e para se expressar, não só utilizando o intelecto, mas todo o corpo, com linguagens verbais e não verbais, com todas as tecnologias disponíveis. Também para o autor (MORAN, 2000), aprende-se melhor com a prática, com experimentação e com a interação com o mundo.

As ferramentas de videoconferência além de transmitirem imagem e áudio, geralmente, possuem outras funcionalidades, entre eles, o quadro branco, transferência de arquivos, bate-papo e gravação. O quadro branco compartilhado é uma área de desenho compartilhada. Permite que os participantes importem imagens gráficas, escrevam, façam esquemas, o que pode esclarecer melhor um assunto. A transferência de arquivos entre os participantes é muito comum em ferramentas de videoconferência. Alguns sistemas permitem que aplicações sejam compartilhadas, permitindo que os participantes utilizem uma aplicação executada no computador de outro participante. $\mathrm{O}$ V. $14 \mathrm{~N}^{\mathrm{o}} 1$, julho, 2016 
bate-papo é útil para registrar perguntas sem interromper um palestrante, para usuários que acompanham a videoconferência e não possuem câmera e microfone interagirem. A função de gravação de conferência permite que o usuário assista novamente uma conferência e, para aqueles que não puderam participar, dá a oportunidade de assistir.

A videoconferência torna-se um recurso poderoso para motivar o aluno. Para que a aprendizagem ocorra é essencial a existência de motivação, pois a aprendizagem é um processo interno que pode ser alimentado e afetado por meio de ações externas, como as interações (RELVAS, 2009). Desta forma, é importante que ambientes virtuais de aprendizagem como o Moodle disponibilizem ferramentas de videoconferência. Apesar do Moodle (MOODLE, 2016) não possuir de forma nativa uma ferramenta de videoconferência, a sua plataforma permite a adição de módulos, denominados plugins. Os módulos permitem adicionar novos recursos e funcionalidades para o Moodle, propiciam, inclusive, que o ambiente seja modelado de acordo com as preferências de cada instituição.

Existem diversas ferramentas de videoconferência disponíveis. Para selecionar quais seriam testadas neste trabalho, o primeiro critério utilizado foi que as mesmas deveriam ter sido implementadas na categoria de software livre. Utilizando este requisito os softwares BigBlueButton (https://github.com/bigbluebutton/bigbluebutton), OpenMeetings(http://openmeetings.apache.org/), Hangouts (https://hangouts.google. com/) e WebHuddle(https://www.webhuddle.com/) tiveram suas principais características analisadas (Tabela 1). A partir desta análise inicial, as ferramentas BigBlueButton (BBB) e OpenMeetings (OM) foram selecionadas para uma avaliação mais criteriosa por possuírem módulo de integração com o Moodle já desenvolvido. As ferramentas Hangouts e WebHuddle foram descartadas.

Tabela 1 - Características das ferramentas de videoconferência

\begin{tabular}{llll|l}
\hline & BigBlueButton & OpenMeetings & WebHuddle & Hangouts \\
\hline Bate-papo & Sim & Sim & Sim & Sim \\
\hline Compartilhamento de Arquivos & Sim & Sim & Sim & Não \\
\hline Quadro Branco & Sim & Sim & Sim & Não \\
\hline Controle de áudio e vídeo por moderador & Sim & Sim & Sim & Sim \\
\hline Limite de pessoas & Não tem & Não tem & Não tem & Limite de 10 \\
\hline Gravação da conferência & Sim & Sim & Sim & Sim \\
\hline Compartilhamento de Tela & Sim & Sim & Sim & Sim \\
\hline Convidar pessoas externas & Não & Sim & Sim & Sim \\
\hline Agenda & Não & Sim & Não & Sim \\
\hline Enquete & Não & Sim & Sim & Não \\
\hline Possui integração com o Moodle & Sim & Sim & Não & Não \\
\hline
\end{tabular}

Além das características apresentadas na Tabela 1, a ferramenta BBB permite a comunicação de vários usuários, sem limite para o número de webcams ativas. As palestras podem ser gravadas no formato AVI (Audio Video Interleave). Nas conferências em grupo, o professor tem o controle de áudio dos microfones de cada um dos participantes, assim como a liberação da webcam. $\mathrm{O}$ aluno pode alterar seu layout para destacar a apresentação, o bate-papo ou o vídeo. No bate-papo, o aluno pode conversar com todos ou ter uma conversa privada com o professor. A aplicação provedora da $\mathrm{BBB}$ fica instalada no servidor da instituição que oferta o serviço.

Já a ferramenta OM é um projeto da Apache. A aplicação provedora do serviço 
pode ser instalada e o acesso dos usuários é feito por um navegador. Com a ferramenta, é possível gerenciar mais de uma instituição, com múltiplos usuários. Estes podem ser organizados em grupos e o administrador do sistema pode atribuir uma sala de conferência a um grupo de usuários, a todos os usuários, ou adicioná-los individualmente. Os usuários podem desenhar no quadro branco, compartilhar as suas telas ou parte delas, gravar a conferência e controlar remotamente outra área de trabalho, mas dependem da permissão do moderador para tais ações. É possível criar uma árvore de documentos para ser compartilhada e uma árvore de acesso privado, desse modo, os arquivos podem ser enviados para as árvores, arrastando-se os e soltando sob a árvore.

\section{Materiais e Métodos}

O principal objetivo deste trabalho foi analisar as ferramentas de videoconferência para verificar qual delas é a mais adequada para o uso no ambiente Moodle. Para alcançá-lo, foi utilizada uma metodologia de pesquisa qualitativa, a qual trabalha com dados descritivos e não estatísticos, não exigindo grande amostra de coleta de dados. As ferramentas $\mathrm{BBB}$ e $\mathrm{OM}$ foram testadas considerando alguns critérios que consideram as características das ferramentas e suas integrações com o Moodle.

Para realizar os testes das ferramentas foram instalados na plataforma Moodle, os módulos do OM e BBB. Estes módulos são utilizados para realizar a comunicação entre cliente-servidor e disponibilizar o processo cliente. $\mathrm{O}$ processo servidor do BBB foi instalado em uma máquina virtual com o sistema operacional Ubuntu. O serviço foi configurado pela aplicação bbb-conf, instalada junto à ferramenta. Como dependências, foram instaladas as aplicações Ffmpeg, LivreOffice e Ruby. O OpenMeetings foi instalado em uma máquina com sistema operacional Windows e para sua base de dados foi utilizado MySql, como seu servidor web foi instalado o Apache e como dependências foram instalados os softwares ImageMagick, GhostScript, SWFTools, JODConverter, OpenOffice, FFMpeg e SoX.

A análise foi realizada utilizando o Método Analítico Hierárquico (MAH), uma ferramenta multicritério utilizado na tomada de decisão (BARAÇAS e MACHADO, 2006). O MAH é atualmente um dos mais respeitados instrumentos para a tomada de decisões multicritério (GUGLIELMETTI, MARINS e SALOMON, 2003). Ele se destaca entre outros métodos por aceitar variáveis quantitativas e qualitativas, de modo que até mesmo impressões subjetivas do tomador de decisão podem ser transformadas em notas lineares para a classificação das alternativas (MORAES e SANTALIESTRA, 2008). Jordão e Pereira (2006) definem o MAH como simples e confiável, facilitando a tomada de decisões entre um número finito de alternativas baseado num conjunto de critérios selecionados com pesos diferentes.

Nesse estudo foi adaptada a estrutura proposta por Jordão e Pereira (2006). A proposta dos autores se destaca pela facilidade de aplicação e a possibilidade de realização dos cálculos e comparações através de matrizes simplificadas. Para isso, a aplicação do MAH é dividida em seis etapas: 1) Definição do Problema; 2) Estruturação Hierárquica do Problema; 3) Construção de Matrizes de Avaliação; 4) Normalização das Matrizes; 5) Construção das Matrizes de Prioridades; 6) Obtenção dos Resultados.

Os critérios para análise (Tabela 2) foram compostos de características desejáveis em uma ferramenta de videoconferência, integrações que possui dentro do Moodle e recursos adicionais que elas dispõem. Levando em consideração que os critérios avaliam características heterogêneas e abordam aspectos diferentes, não há como compará-los entre si, portanto eles receberam o mesmo peso. Entretanto, como o foco deste trabalho é avaliar a integração com o Moodle, os critérios referentes ao módulo de integração receberam um peso maior.

V. $14 \mathrm{~N}^{\mathrm{o}} 1$, julho, 2016 
Para a avaliação, foram definidos níveis de atendimento da ferramenta em relação ao critério. São quatro esses níveis: (a) atendimento pleno, quando a ferramenta dispõe de todos os itens descritos no critério; (b) atendimento parcial, quando algum item do critério não foi atendido; (c) atendimento mínimo, quando a ferramenta não atende a mais de um item do critério ou atende pouco a exigência do critério (d) inexistente quando a ferramenta não atende de nenhuma forma o critério, ou que possui relativo ao critério é irrelevante.

Tabela 2 - Critérios para Análise das Ferramentas

\begin{tabular}{|c|c|c|c|}
\hline ID & Peso & Critério & Itens analisados \\
\hline $\mathrm{C} 1$ & 1 & Bate-papo & $\begin{array}{l}\text { Dispõe de conversas abertas e privadas, permite uso de cores e } \\
\text { emotions. }\end{array}$ \\
\hline $\mathrm{C} 2$ & 1 & Quadro branco & $\begin{array}{l}\text { Possui quadro branco que permite desenhar, escrever, fazer } \\
\text { esquemas e importar imagens. Permite que participantes (não } \\
\text { moderadores) utilizem o quadro e que dois ou mais utilizem ao } \\
\text { mesmo tempo. }\end{array}$ \\
\hline $\mathrm{C} 3$ & 1 & Apresentação de arquivos & $\begin{array}{l}\text { Possui recurso para apresentar arquivos do Power Point, do Word } \\
\text { e de formato PDF. }\end{array}$ \\
\hline $\mathrm{C} 4$ & 1 & Transferência de arquivos & Permite transferir arquivos. \\
\hline \multirow[t]{2}{*}{ C5 } & 1 & $\begin{array}{l}\text { Gravação da } \\
\text { videoconferência }\end{array}$ & É possível gravar a conferência. \\
\hline & 1 & Compartilhamento de tela & $\begin{array}{l}\text { O moderador e participantes podem compartilhar a suas telas e é } \\
\text { possível dar o controle da tela a outros. }\end{array}$ \\
\hline $\mathrm{C} 7$ & 1 & Limite de participantes & Suporta 30 participantes. \\
\hline $\mathrm{C} 8$ & 1 & $\begin{array}{l}\text { Controle de áudio e vídeo } \\
\text { por moderador }\end{array}$ & $\begin{array}{l}\text { Existe um papel de moderador para áudio e vídeo e ele pode ser } \\
\text { transferido a outra pessoa na conferência. }\end{array}$ \\
\hline C9 & 1 & Agendar conferência & $\begin{array}{l}\text { Existe recurso para realizar agendamento e participantes recebem } \\
\text { notificações sobre alterações no evento. }\end{array}$ \\
\hline $\mathrm{C} 10$ & 1 & Enquete & Possui recurso para enquetes durante a conferência. \\
\hline $\mathrm{C} 11$ & 9 & $\begin{array}{l}\text { Integração de cadastro de } \\
\text { usuários }\end{array}$ & $\begin{array}{l}\text { Dispõe de integração do seu cadastro de usuários com o cadastro } \\
\text { do Moodle }\end{array}$ \\
\hline $\mathrm{C} 12$ & 9 & Integração de bate-papo & $\begin{array}{l}\text { Permite utilizar somente o bate-papo da ferramenta, sem } \\
\text { videoconferência, dentro do Moodle. }\end{array}$ \\
\hline $\mathrm{C} 13$ & 9 & $\begin{array}{l}\text { Integração de cursos e } \\
\text { grupos }\end{array}$ & $\begin{array}{l}\text { Ao ser criada uma videoconferência, a ferramenta considerar } \\
\text { automaticamente como participantes os alunos de um mesmo } \\
\text { curso ou grupo e, posteriormente, participantes podem ser } \\
\text { adicionados ou removidos. }\end{array}$ \\
\hline $\mathrm{C} 14$ & 9 & Integração de agenda & $\begin{array}{l}\text { Possui integração com a agenda nativa do Moodle, sendo possível } \\
\text { agendar uma videoconferência através dela. }\end{array}$ \\
\hline $\mathrm{C} 15$ & 9 & Integração de enquete & $\begin{array}{l}\text { Possui integração com a ferramenta Escolha do Moodle sendo } \\
\text { possível utilizá-la durante a videoconferência. }\end{array}$ \\
\hline $\mathrm{C} 16$ & 9 & $\begin{array}{l}\text { Integração de } \\
\text { compartilhamento de } \\
\text { arquivos }\end{array}$ & $\begin{array}{l}\text { Permite compartilhar arquivos já existentes no ambiente Moodle e } \\
\text { arquivos compartilhados na videoconferência podem ser salvos e } \\
\text { disponibilizados aos alunos, manual ou automaticamente, no } \\
\text { ambiente. }\end{array}$ \\
\hline $\mathrm{C} 17$ & 9 & $\begin{array}{l}\text { Acesso por Dispositivos } \\
\text { Móveis }\end{array}$ & $\begin{array}{l}\text { A conferência pode ser feita através de dispositivos móveis sem } \\
\text { deformar a interface. }\end{array}$ \\
\hline
\end{tabular}


Os testes realizados no OP mostraram que os critérios de bate-papo $(\mathrm{C} 1)$, quadro branco (C2), gravação da videoconferência (C5), compartilhamento de tela (C6), limite de participantes (C7), controle de áudio e vídeo por moderador (C16), enquete (C10) e integração de cadastro de usuários (C11) foram atendidos plenamente pela ferramenta.

Os critérios apresentação de arquivos (C3), integração de bate-papo (C12), e integração de cursos e grupos foram atendidos parcialmente. OM permite que arquivos no formato PDF possam ser carregados, porém não é possível carregar documentos do Word ou PowerPoint. A OM não possui configuração para utilizar uma sala somente para bate-papo e mesmo sem nenhum quadro branco, a área dele ainda ficará reservada. Contudo, se o moderador bloquear o recurso de vídeo, poderá ser utilizado somente o bate-papo.

Os critérios transferência de aquivos (C4), agendar conferência (C9), integração de agenda (C14), integração de enquete (C15), integração de compartilhamento de arquivos (C16) e acesso por dispositivos móveis (C17) não foram atendidos. Os arquivos carregados não podem ser baixados pelos alunos e nem pelos moderadores, visto que não existe recurso para transferência de arquivos. De igual modo, não é possível carregar no quadro branco arquivos existentes no Moodle, nem disponibilizar arquivos da videoconferência no ambiente. A atividade OM não possui integração com a agenda nativa do Moodle e nem possui recurso para agendamento de videoconferência. Somente é possível usar a agenda para cadastrar um evento com informações da videoconferência, porém sem vínculo com a atividade. Uma enquete por vez pode ser criada no OM, mas também não existe integração com o moodle. A aplicação ao ser acessada por dispositivo móvel, não carregou usuários logados, não mostrou o conteúdo do quadro branco. Também não foi possível utilizar o bate-papo nem microfone e vídeo, seu zoom distorcia a interface e não se ouvia os participantes da conferência, apesar de o navegador possuir permissão para utilização de microfone e câmera do celular.

Já os testes realizados no BBB mostraram que os critérios apresentação de arquivos (C3), gravação da videoconferência (C5), limite de participantes (C7), agendar conferência (C9), integração de cadastro de usuários (C11), integração de cursos e grupos $(\mathrm{C} 13)$ e integração de agenda $(\mathrm{C} 14)$ foram atendidos plenamente pelo software. Os critérios relativos ao bate-papo $(\mathrm{C} 1)$, quadro branco $(\mathrm{C} 2)$, compartilhamento de tela (C6), controle de áudio e vídeo por moderador (C8) foram parcialmente atendidos. O bate-papo não tem recursos de cores, emotions, estilos de fonte ou cor de fundo para diferenciar as mensagens e nem captura do histórico, mas nele é possível definir o tamanho da fonte, sendo 18 o tamanho máximo. No quadro branco não é possível selecionar, mover, editar ou apagar um desenho e nem suporta a edição por mais de uma pessoa. Existem somente as funcionalidades de desfazer o último desenho, limpar todo o quadro e transferir o controle para outro participante. O compartilhamento de tela funciona apenas com Firefox, e somente para o primeiro moderador logado, não podendo ser, de modo algum, transferido a outro moderador ou dado a um aluno. $\mathrm{O}$ moderador pode bloquear o microfone dos estudantes, habilitado por padrão, mas não tem controle sobre o vídeo.

Os critérios integração de bate-papo (C12) e acesso por dispositivos móveis (C17) são atendidos de forma mínima. Não há opção para criar uma sala somente com o bate-papo. É possível alterar o layout da tela destacando apenas o bate-papo, mas o layout é uma escolha individual, cada aluno escolhe o seu, inclusive podendo minimizar qualquer área da sala. A BBB sendo acessada por dispositivo móvel não deforma sua tela e a utilização de zoom funciona adequadamente, é possível utilizar o bate-papo, acompanhar o conteúdo do quadro branco e também o compartilhamento de tela. Porém não é possível ouvir os participantes nem utilizar microfone e vídeo, ao habilitar o microfone a tela se fecha, e o botão para compartilhar vídeo fica desabilitado, apesar de V. $14 \mathrm{~N}^{\mathrm{o}} 1$, julho, 2016 
o navegador ter permissão para utilização de microfone e câmera do celular.

O BBB não dispõe de recursos para enquete, e para a transferência de arquivos, não atendendo os critérios 4,10,16 e 17. Um resumo do atendimento dos critérios pode ser observado na tabela 3

Tabela 3 - Tabela geral do cruzamento entre ferramentas e critérios

\begin{tabular}{|c|c|c|c|c|c|}
\hline Critérios & OM & BBB & Critéri & OM & BBB \\
\hline $\mathrm{C} 1$ & Pleno & Parcial & C10 & Pleno & Não atende \\
\hline $\mathrm{C} 2$ & Pleno & Parcial & C11 & Pleno & Pleno \\
\hline $\mathrm{C} 3$ & Mínimo & Pleno & $\mathrm{C} 12$ & Mínimo & Mínimo \\
\hline $\mathrm{C} 4$ & Não atende & Não atende & C13 & Mínimo & Pleno \\
\hline $\mathrm{C} 5$ & Pleno & Pleno & C14 & Não atende & Pleno \\
\hline$\overline{\mathrm{C} 6}$ & Pleno & Parcial & $\mathrm{C} 15$ & Não atende & Não atende \\
\hline $\mathrm{C} 7$ & Pleno & Pleno & C16 & Não atende & Não atende \\
\hline $\mathrm{C} 8$ & Pleno & Parcial & C17 & Não atende & Mínimo \\
\hline
\end{tabular}

\section{Resultados Obtidos}

Após a realização dos testes nas ferramentas integradas ao Moodle e a verificação do nível de atendimento delas para cada critério, esta seção traz a comparação das ferramentas entre si. As ferramentas serão comparadas por meio de matrizes conforme é estabelecido no MAH. Quando um critério linha é mais importante do que um critério coluna, a célula de cruzamento dos dois terá valores de 2 até $9(2=$ pouquíssimo mais importante a 9 = extremamente importante). No caso contrário, se o critério linha é menos importante que o critério coluna, então, o cruzamento terá valores de 1/2 até 1/9 $(1 / 2=$ pouquíssimo menos importante a $1 / 9=$ extremamente menos importante $)$. No caso de um critério ter a mesma importância que o outro, o cruzamento receberá peso 1 , assim, também quando um critério é comparado com ele mesmo o valor será 1 . A Tabela 4 demonstra os valores da Escala de Saaty (2013) juntamente aos seus inversos.

Tabela 4 - Escala de Saaty Complementada

\begin{tabular}{|c|c|c|c|c|c|c|c|c}
\hline $1 / 9$ & $1 / 7$ & $1 / 5$ & $1 / 3$ & 1 & 3 & 5 & 7 & 9 \\
\hline $\begin{array}{c}\text { Extremament } \\
\text { e }\end{array}$ & Muito & Moderadamente & Pouco & Igual & Pouco & Moderadamente & Muito & Extremamente \\
\hline \multicolumn{3}{c}{ Menos importante } & \multicolumn{5}{c}{ Mais importante } \\
\hline
\end{tabular}

Fonte: Pappa(2012)

Para cada critério é criada uma matriz, esta conterá o cruzamento entre os níveis de atendimento de cada ferramenta. As comparações foram feitas definindo a importância do atendimento da ferramenta da linha em relação ao atendimento da ferramenta da coluna. A Tabela 5 representa a matriz de avaliação para os critérios $\mathrm{C} 1$, C2, C6 e C8. Esses critérios foram plenamente atendidos pela $\mathrm{OM}$ e parcialmente atendidos pela $\mathrm{BBB}$. $\mathrm{O}$ atendimento é parcial para o critério $\mathrm{C} 1$, devido a $\mathrm{BBB}$ não apresentar recurso de cores e emotions em seu bate-papo, com relação ao critério $\mathrm{C} 2 \mathrm{o}$ quadro branco da BBB não suporta a edição por mais de uma pessoa ao mesmo tempo. Pela BBB não dispor de controle remoto de tela, atente parcialmente o critério C6 e pelo fato do moderador não ter controle sobre o vídeo dos participantes, também atende parcialmente o critério $\mathrm{C} 8$.

V. $14 \mathrm{~N}^{\circ} 1$, julho, 2016 
A BBB teve melhor desempenho no critério C3 (Tabela 6), atendendo ele plenamente, enquanto a $\mathrm{OM}$ o atendeu minimamente, por não suportar documentos do Word e PowerPoint em seu quadro branco.

Tabela 5 - Critérios C1,C2,C6 e C8

\begin{tabular}{l|c|c|c}
\hline & OM & BBB & Média \\
\hline OM & 1 & 5 & 0,83 \\
\hline BBB & $1 / 5$ & 1 & 0,17 \\
\hline Soma & 1,2 & 6 & \\
\hline
\end{tabular}

Tabela 6 - Critério C3

\begin{tabular}{l|c|c|c}
\hline & OM & BBB & Média \\
\hline OM & 1 & $1 / 7$ & 0,12 \\
\hline BBB & 7 & 1 & 0,87 \\
\hline Soma & 8 & 1,14 & \\
\hline
\end{tabular}

A avaliação dos critérios $\mathrm{C} 4, \mathrm{C} 5, \mathrm{C} 7, \mathrm{C} 11, \mathrm{C} 12, \mathrm{C} 15$ e C16 é representada pela Tabela 7. Nestes critérios a BBB e a OM apresentaram o mesmo nível de atendimento. Ambas atendem plenamente os critérios C5 e C7, suportando mais de 30 participantes em suas conferências, o $\mathrm{C} 11$, pois utilizam os cadastros de usuários do Moodle. Elas não atendem os critérios $\mathrm{C} 4$, C15 e C16, consequentemente, por não implementarem a transferência de arquivos de forma funcional, por não possuírem integração de enquete e integração de compartilhamento de arquivos. Apresentam atendimento mínimo para o critério $\mathrm{C} 12$, por não possuírem recursos para a criação de salas somente para bate-papo, de forma satisfatória.

A BBB se sobressaiu nos critérios C9 e C14, referentes a agendamento das videoconferências, atendendo-os plenamente enquanto a OM não os atendeu (Tabela 8). O contrário acontece com o critério C10 (Tabela 9), atendido plenamente pela OM e não atendido pela $\mathrm{BBB}$ por não possuir recurso de enquete.

Tabela 7 - Critérios C4, C5, C7, C11, C12, C15 e

Tabela 8 - Critérios C9 e C14

$$
\mathrm{C} 16
$$

\begin{tabular}{l|c|c|c}
\hline & OM & BBB & Média \\
\hline OM & 1 & 1 & 0,5 \\
\hline BBB & 1 & 1 & 0,5 \\
\hline Soma & 2 & 2 & \\
\hline
\end{tabular}

\begin{tabular}{l|c|c|c}
\hline & OM & BBB & Média \\
\hline OM & 1 & $1 / 9$ & 0,1 \\
\hline BBB & 9 & 1 & 0,9 \\
\hline Soma & 10 & 1,11 & \\
\hline
\end{tabular}

A BBB também mostrou melhor desempenho nos critérios $\mathrm{C} 13$ e $\mathrm{C} 17$. O critério C13 (Tabela 10) foi atendido minimamente pela OM, por não dispor de integração com os grupos do Moodle, entretanto foi atendido plenamente pela BBB. O critério C17 (Tabela 11) foi atendido minimamente pela BBB, por alguns de seus recursos funcionarem em dispositivos móveis, enquanto o mesmo não foi atendido pela $\mathrm{OM}$, por não apresentar nenhum recurso funcional em dispositivos móveis.

Tabela 9 - Critério 10

\begin{tabular}{l|c|c|c}
\hline & OM & BBB & Média \\
\hline OM & 1 & 9 & 0,9 \\
\hline BBB & $1 / 9$ & 1 & 0,1 \\
\hline Soma & 1,11 & 10 & \\
\hline
\end{tabular}

Tabela 10 - Critério C13

\begin{tabular}{l|c|c|c}
\hline & OM & BBB & Média \\
\hline OM & 1 & $1 / 7$ & 0,12 \\
\hline BBB & 7 & 1 & 0,87 \\
\hline Soma & 8 & 1,14 & \\
\hline
\end{tabular}


Tabela 11 - Critério C17

\begin{tabular}{|c|c|c|c}
\hline & OM & BBB & Média \\
\hline OM & 1 & $1 / 7$ & 0,12 \\
\hline BBB & 7 & 1 & 0,87 \\
\hline Soma & 8 & 1,14 & \\
\hline
\end{tabular}

Após as matrizes dos 17 critérios criadas, foram calculadas as notas finais de cada ferramenta considerando os pesos dos critérios, estas são apresentadas na Tabela 12. Para melhor comparação o quadro ainda mostra os níveis de atendimento das duas ferramentas, lado a lado, seguidos das pontuações, para cada um dos critérios. A BBB atendeu plenamente a seis critérios, parcialmente também a seis, minimamente a dois critérios, e não atendeu a quatro. A $\mathrm{OM}$ atendeu plenamente a sete critérios, parcialmente somente a dois, minimamente a três e os critérios não atendidos foram seis. O resultado final apresenta a BBB com maior pontuação, 44,73, tendo a OM obtido a nota 28,17 .

Tabela 12 - Pontuação Final das Ferramentas

\begin{tabular}{|c|c|c|c|c|}
\hline & Critério & Peso & $\mathbf{O M}$ & BBB \\
\hline $\mathrm{C} 1$ & Bate-papo & 1 & 0,83 & 0,17 \\
\hline $\mathrm{C} 2$ & Quadro branco & 1 & 0,83 & 0,17 \\
\hline $\mathrm{C} 3$ & Apresentação de arquivos & 1 & 0,12 & 0,87 \\
\hline $\mathrm{C} 4$ & Transferência de arquivos & 1 & 0,5 & 0,5 \\
\hline $\mathrm{C} 5$ & Gravação da videoconferência & 1 & 0,5 & 0,5 \\
\hline C6 & Compartilhamento de tela & 1 & 0,83 & 0,17 \\
\hline $\mathrm{C} 7$ & Limite de participantes & 1 & 0,5 & 0,5 \\
\hline $\mathrm{C} 8$ & Controle de áudio e vídeo por moderador & 1 & 0,83 & 0,17 \\
\hline C9 & Agendar conferência & 1 & 0,1 & 0,9 \\
\hline $\mathrm{C} 10$ & Enquete & 1 & 0,9 & 0,1 \\
\hline $\mathrm{C} 11$ & Integração de cadastro de usuários & 9 & 4,5 & 4,5 \\
\hline $\mathrm{C} 12$ & Integração de bate-papo & 9 & 4,5 & 4,5 \\
\hline $\mathrm{C} 13$ & Integração de cursos e grupos & 9 & 1,08 & 7,83 \\
\hline C14 & Integração de agenda & 9 & 0,9 & 8,1 \\
\hline $\mathrm{C} 15$ & Integração de enquete & 9 & 4,5 & 4,5 \\
\hline C16 & Integração de compartilhamento de arquivos & 9 & 4,5 & 4,5 \\
\hline $\mathrm{C} 17$ & Acesso por Dispositivos Móveis & 9 & 2,25 & 6,75 \\
\hline \multicolumn{3}{|c|}{ Pontuação Total } & 28,17 & 44,73 \\
\hline
\end{tabular}

\section{Conclusão}

Considerando o fato do Moodle não dispor nativamente de videoconferência, este artigo se propôs a avaliar as ferramentas de videoconferência BBB e OM, para pontuar suas 
características e verificar qual é a mais apta à integração com o Moodle. Pelos testes das aplicações pode-se constatar que a BBB e a OM possuem ótimos recursos e partilham de muitas funções semelhantes, ambas possuem características básicas de uma fermenta de videoconferência, como quadro branco, bate-papo, gravação da conferência e o papel de moderador e podem ser utilizadas para videoconferências integradas ao Moodle, ou de forma independente. A OpenMeetings possui o recurso de enquete e controle remoto de tela, não presentes na BigBlueButton, entretanto esta última se destacou por oferecer um maior nível de integração com o Moodle. Pontos que fazem a BBB se sobressair são a integração com os grupos de usuários do ambiente Moodle, que possibilita criar uma sala de videoconferência para cada grupo automaticamente, a integração com a agenda do Moodle, e também por apresentar melhor desempenho, mesmo que falho, ao ser acessada por dispositivos móveis. Apesar deste artigo não ter incluído a interface da ferramenta como um critério da avaliação, vale salientar que a BBB possui uma interface mais limpa, com aspecto mais moderno e profissional, além de possuir um layout personalizável.

\section{Referências Bibliográficas}

ALAVA, Seraphin et al. Ciberespaço e formações abertas: rumo a novas práticas educacionais? Porto Alegre: Artmed, 2002.

BARAÇAS, F. J. L.; MACHADO, J. P. A. A Análise Multicritério na Tomada de Decisão - o Método Analítico Hierárquico de T. L. Saaty. 2006. Instituto Politécnico de Coimbra. Departamento de Engenharia Civil, 2006.

GUGLIELMETTI, Fernando Ribeiro; MARINS, Fernando Augusto Silva; SALOMON, Valério Antonio Pamplona. Comparação teórica entre métodos de auxílio à tomada de decisão por múltiplos critérios. In: XXIII Encontro Nac. de Eng. de Produção - Ouro Preto, MG, Brasil, 21 a 24 de out de 2003.

JORDÃO, Bruno Miguel da Cruz. PEREIRA, Susete Rodrigues. A Análise Multicritério na Tomada de Decisão - O Método Analítico Hierárquico de T. L. Saaty: Desenvolvimento do método com recurso à análise de um caso prático explicado ponto a ponto. 2006. Dissertação (Gestão de Empreendimentos) - Departamento de Engenharia Civil, Instituto Politécnico de Coimbra.

MOODLE. Open-source learning platform. 2016. Disponível em: <https://moodle.org/>. Acesso em: 1 março 2016.

MORAES, Edmilson Alves; SANTALIESTRA, Rodrigo. Modelo de decisão com múltiplos critérios para escolha de software de código aberto e software de código fechado. Organizações em contexto, Ano 4, n. 7, junho 2008.

MORAES, Maria Cândida (Org.). Educação a distância: fundamentos e práticas. São Paulo: Unicamp/NIED, 2002.

MORAN COSTAS, José Manuel. Ensino e aprendizagem inovadores com tecnologias. Informática na Educação: Teoria \& Prática. Porto Alegre, vol. 3, set. 2000.

MORAN COSTAS, José Manuel. Mudanças na Comunicação Pessoal: gerenciamento integrado da comunicação pessoal, social e psicológica. São Paulo: Paulinas, 1998.

MÜLBERT, Ana Luisa; GIRONDI, Ariane; PEREIRA, Alice T. Cybis; NAKAYAMA, Marina K. A interação em ambientes virtuais de aprendizagem: motivações e interesses dos alunos. 2011. Disponível em: <http://seer.ufrgs.br/ index.php/renote/article/view/21972/12745>. Acesso em: 28 mar. 2015.

PAPPA, Márcia Fernanda. Aplicação da Metodologia AHP na Hierarquização dos Critérios de Quailidade de Trânsito das Cidades.2012. 100 f. Dissertação (Mestrado) - Curso de Engenharia Urbana, Universidade Estadual de Maringá, Maringá, 2012.

SAATY, Thomas Lorie; VARGAS Luis González. Decision Making with the Analytic Network Process: Economic, Political, Social and Technological Applications with Benefits, Opportunities, Costs and Risks. New York: Springer Science \& Business Media, 2013.

SILVA, Adelina Maria Pereira. Processos de ensino-aprendizagem na era digital. O Professor, Portugal, n.93. Alfragide: Caminho, mai.-ago., 2006. 